\title{
AUGUSTIN ANASSE
}

Université de Bouaké (Côte d'lvoire)

\section{MARC BIDAN}

Université de Nantes (France)

\section{ALIDOU OUEDRAOGO}

Université de Moncton (Canada)

\section{GWENAËLLE ORUEZABALA}

Université de Nantes (France)

\section{JEAN-MICHEL PLANE}

Université Paul-Valéry Montpellier 3 (France)

\section{Alternatives africaines en management Entre frugalité et agilité}

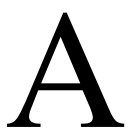

« l'ombre d'un caïlcedrat géant de Bamako, on trouvait encore, il y a quelques années, un bricoleur malin que les experts des Nations unies appelaient le docteur Mobylette. Chaque matin de bonne heure, il installait sa caisse à outils au pied de l'arbre. Le bonhomme avait calculé qu'avec 2000 francs CFA (3 euros) par jour, il pouvait facilement subvenir à ses besoins comme à ceux de sa famille. Une fois la somme empochée, il cessait de travailler, son frère prenant le relais sous le caiilcedrat. Après l'avoir longuement observé, quelques spécialistes du développement proposèrent au docteur Mobylette de voir les choses en plus grand, d'ouvrir un atelier dans la capitale, d'adopter des horaires fixes, et, au bout du compte, de se retrouver à la tête d'une véritable entreprise. Le docteur Mobylette mit peu de temps pour donner sa réponse. Il remercia mais refusa, estimant qu'un tel chambardement dans l'usage de la caisse à outils reviendrait à voler le travail de son frère. » (Fottorino et al., 1992). 
Cette conception de la gestion de l'entreprise par le docteur Mobylette, largement partagée et comprise en Afrique, peut paraître incongrue et déstabilisante dans certains contextes. En l'occurrence dans le contexte occidental, certains se demandent si «L'entreprise africaine » existe vraiment ? Si l'on peut raisonnablement tenter d'en cerner ses contours et son management souvent invisible ? L'invisibilité du management, l'existence de variables « irrationnelles », une gestion par l'ambiguïté et des relations sociales complexes et parfois ambivalentes... tels sont quelques traits caractéristiques résultant d'observations directes et/ou participantes réalisées par les auteurs de ce dossier spécial pendant plus d'une dizaine d'années. Pour autant, existe-t-il des alternatives africaines en management ? Comment peut-on les identifier? Sont-elles crédibles et dans quelle mesure peuvent-elles être transposables d'un contexte à un autre ? Peuvent-elles apporter des solutions novatrices en ces temps extrêmement troublés par la pandémie de la Covid-19 ? Confrontée à une crise sanitaire sans précédent depuis la Seconde Guerre mondiale, à des défis de plus en plus complexes et variés et face aux économies occidentales et asiatiques encore dominantes mais désormais ébranlées, l'Afrique subsaharienne développe des stratégies entrepreneuriales affirmées, souvent originales. La crise sanitaire liée à la Covid-19 que le monde traverse conduira probablement à un changement de raisonnement voire de paradigme quant à la mondialisation : on ira peut-être dans le sens de la démondialisation et de la réindustrialisation : les relations entre le monde occidental, l'Asie et l'Afrique s'en trouveront inévitablement bouleversées ne serait-ce que du point de vue des chaînes logistiques qui pourraient peut-être se rapprocher encore davantage entre l'Europe et l'Afrique. Au total, la résilience qui résultera de cette crise sans précédent impactera aussi bien les écosystèmes d'affaires que les méthodes de management qui devront certainement apporter des réponses plus originales. Ces questions et bien d'autres se posent avec d'autant plus d'acuité car le continent africain connaît l'essor d'un véritable écosystème entrepreneurial dynamique qui constitue simultanément un outil durable de création d'emplois et d'activités mais aussi un ressort essentiel au développement. De façon générale, la dynamique économique et entrepreneuriale en Afrique subsaharienne est de plus en plus spectaculaire, médiatisée, ancrée mais aussi variée. Par exemple, selon le rapport 2017 «Women's Entrepreneurship » produit par le GEM (Global Entrepreneurship Monitor), l'Afrique est devenu le premier continent de l'entrepreneuriat féminin avec une proportion de $27 \%$ de femmes qui créent et développent des entreprises. De façon surprenante, on observe que $70 \%$ de ces femmes ne bénéficient d'aucun financement. Une telle dynamique entrepreneuriale est difficile à analyser tant les dimensions cachées et originales semblent importantes et finalement à ce jour peu discutées. En ce sens, le rapport du GEM 2017 précise que trois pays sortent du lot. Il s'agit du Kenya, du Nigeria mais aussi de l'Afrique du Sud, pays parfois appelés « start-up nations » qui recueillent la plupart des 560 millions de dollars investis dans ces nouvelles formes d'entreprises fondées sur des principes organisationnels originaux.

Malgré l'attrait de terrains de recherche potentiellement féconds en Afrique 
subsaharienne - et en particulier s'agissant d'organisations de petite taille - les chercheurs en gestion ont délaissé pendant longtemps ce champ d'investigation (Tidjani et Kamdem, 2010). Depuis plusieurs années, une communauté d'enseignantschercheurs francophones s'est progressivement constituée à la suite de taux de succès plus élevés aux différents concours de l'agrégation en sciences de gestion en particulier durant les deux dernières décennies. Les contributions que l'on observe de plus en plus sur «l'entreprise africaine » (par exemple Worou, 2011; Nkakleu, 2016 ; Hounkou, 2015) révèlent souvent une « prééminence de pratiques informelles socialement ancrées dans la culture communautaire » et la présence de pratiques de gestion du personnel hybrides (Nyobe et Plane, 2020) même si le modèle arbitraire fondé sur le mode informel, des critères intuitifs ou implicites, une formation sur le tas et un management autoritaire et/ou paternaliste semble l'emporter sur le modèle objectivant du point de vue des pratiques $\mathrm{RH}$ en particulier au sein de petites entreprises (Pichault et Nizet, 2000). Nonobstant, ces entreprises africaines sont aussi capables d'innovations organisationnelles, de flexibilité et de performances étonnantes dans des domaines d'activités assez variés (télécommunications, secteur minier, secteur des services et du divertissement, etc.). En effet, les agents économiques le plus souvent partent de ce qu'ils ont pour élaborer chemin faisant une aventure entrepreneuriale. Il se passe quelque chose de très particulier, de spécifique et de passionnant à relever en Afrique subsaharienne sur le plan notamment de l'entrepreneuriat effectual (Sarasvathy, 2001). Les considérations qui suivent et ce dossier consacré à
« l'entreprise africaine » tendent à étayer la thèse selon laquelle il s'agit effectivement d'étudier «ce quelque chose de très particulier» sous l'angle des innovations frugales et parfois des ruptures (ou innovations radicales) que l'on peut observer. L'objet de recherche ne serait donc pas le culturalisme, ni même l'institutionnalisme mais plutôt des situations de management contextualisées et ancrées dans des réalités locales. La finalité serait d'essayer de dévoiler et d'expliquer des mécanismes complexes conduisant à des équilibres sociaux ou à des performances pouvant être assez variables. L'effectuation apporte également un nouvel éclairage sur le frugalisme et sur la manière de concevoir le processus de décision et l'agilité de l'entrepreneur africain (voir l'entretien réalisé avec S. Sarasvathy proposé dans ce dossier). Ce dernier fixe ses objectifs en fonction de ses moyens souvent limités et de ce qu'il est vraiment. C'est probablement en essayant de décortiquer ce processus assez opaque que l'on comprendra mieux cette façon de réussir et son cheminement. En effet, la plupart des dirigeants africains réussissent sans avoir suivi le processus dit linéaire et classique de création et de développement d'une affaire (étude de marché, business plan, etc.). Le processus que l'on observe est beaucoup plus heuristique, plus personnel également et est fondé sur des ressources plus ou moins disponibles que le dirigeant est capable de mobiliser ainsi que sur les opportunités qui se présentent. Ce dossier spécial a aussi pour objectif de présenter et d'investiguer cette dynamique et d'en souligner les dimensions cachées et alternatives en particulier sur le plan de la gestion et du management. Au fond, quelles leçons peut-on tirer du management en contexte des entreprises africaines de petite 
taille ? Que valent ces connaissances sur le plan épistémologique et dans quelle mesure peuvent-elles être transposables et enseignables auprès de publics variés ? Quels sont les concepts et les mécanismes originaux qui semblent ressortir des situations de gestion africaines observées sur un terrain souvent opaque mais complexe, riche et captivant? Ce dossier cherche également, au travers des quatre articles proposés, à contribuer à une meilleure compréhension des réalités de terrain vécues par les acteurs généralement associés à l'entreprise africaine et ainsi de souligner l'intérêt d'un enrichissement des connaissances dans ce domaine. Sur le plan managérial, le but recherché pour les auteurs est de fournir à ces acteurs des pistes de réflexion et des leviers d'action importants pour contribuer au développement de la performance de ces entreprises. En ce sens, cet article introductif cherche à élaborer un état des lieux quant aux réflexions menées par les chercheurs sur cette problématique, tout en proposant plusieurs éclairages permettant de mieux agir en situation de gestion. Ainsi, dans la première partie de cet article, deux approches structurant les recherches dans le champ investi, sont mises en évidence. Dans une seconde partie, et avant de présenter les contributions à ce dossier, nous partirons à la recherche d'alternatives africaines en management.

\section{I - ÉTAT DES APPORTS DES CHERCHEURS EN SCIENCES DE GESTION SUR « L'ENTREPRISE AFRICAINE »}

Sur le plan théorique, la question de l'applicabilité des approches et des pratiques globales de management dans les entreprises africaines a souvent été une préoccupation pour les chercheurs qui s'intéressent aux pays émergents (Hoskisson et al., 2000). Dans le contexte d'une grande diversité culturelle et de la mondialisation, deux perspectives ont souvent été opposées : la convergence et la divergence. La première postule l'existence de modèles universels de gestion transcendant les frontières et mis en œuvre par assimilation. On est dans la perspective d'un modèle universaliste suivant lequel il existe des prescriptions standard applicables, assimilables et transposables à tous les contextes. La seconde est la perspective bien connue déjà de la contingence. Elle présente deux visions. Dans la première, plus culturaliste, les entreprises africaines doivent puiser dans leurs valeurs et leur organisation sociétale, pour ériger les fondements d'un management africain propre (Bourgoin, 1984 ; Delalande, 1987 ; Kamdem, 2002 ; Hernandez et Kamdem, 2007). L'idée de bâtir un management africain singulier et distinctif est séduisante mais elle semble ignorer que la culture est une variable dynamique et donc qu'elle évolue au contact avec les autres types d'environnement. La seconde vision postule que le management à l'occidentale doit s'adapter et se transformer dans le contexte africain, dans une sorte d'hybridation ou de métissage entre les méthodes occidentales et les valeurs africaines. Dans le prolongement de cette vision, Kiggundu et al. (1983) ont effectué une revue comparative des recherches portant sur les théories du management. Ils y indiquent que dans le processus de transfert de compétences, quand le noyau technique de l'organisation en constitue le point central, le transfert des théories des pays développés vers ceux en développement ne pose généralement 
aucun problème. En revanche, lorsque le point central du transfert de compétences vise une relation entre l'organisation et son environnement, l'application des théories occidentales rencontre de sérieuses résistances. Les différences et les décalages culturels semblent incontournables et les gestionnaires doivent inévitablement s'adapter aux spécificités et à la culture locale. Trancher entre ces deux perspectives est loin d'être aisé et dépend probablement du projet de recherche (applicabilité des pratiques de gestion occidentales versus appropriation des théories de la gestion). En ce sens, Hafsi et Farashashi (2005) montrent que les approches occidentales du management s'appliquent de plus en plus dans les entreprises africaines (apport de la théorie de la convergence) notamment à cause des pressions institutionnelles induites par la mondialisation et par les organisations internationales telles que le FMI, la Banque mondiale ou encore les agences de l'ONU. Ces pressions de facto et de jure s'incarnent sous forme de normes, de lois et de règlements qui s'imposent à toutes les formes d'entreprises. Ainsi, les particularités de l'environnement local tendent à s'estomper face aux lois, normes et processus internationaux qui s'imposent à tous (apport de la théorie institutionnelle).

\section{1. « L'entreprise africaine » par les concepts de frugalité et d'agilité}

Sur le plan conceptuel, plusieurs concepts sont, ici, mobilisés à titre indicatif. D'abord, celui de la frugalité qui a émergé récemment dans la littérature académique mais qui semble correspondre à des pratiques liées à la simplicité et à la sobriété répandues depuis longtemps dans différentes régions du monde. Parler de frugalité consiste à admettre l'idée stimulante qu'il est possible de «faire mieux avec moins ». Plusieurs publications ayant connu un large succès, en particulier les ouvrages de Radjou et al. (2013) et de Radjou et Prabhu (2015) sur l'innovation frugale, ont fortement contribué à la popularité de ce concept et souligné l'intérêt de «savoir et pouvoir faire mieux avec moins ». Généralement, ces travaux sur les innovations frugales sont abondamment cités. Appliqués à des situations de management africaines, il est aussi possible d'avancer l'idée d'innovation inversée dans le sens où les pays en développement peuvent aussi apporter une innovation dans un pays développé.

La réflexion sur les innovations frugales et les observations que l'on peut faire en Afrique subsaharienne permettent de parler de la «débrouillardise » (« Jugaad » suivant le terme indien) dès lors que l'on est dans le cadre d'un produit moins cher que l'on met sur le marché et qui présente la caractéristique d'être fondé davantage sur "l'ingéniosité et le bon sens que sur le progrès technologique ». En Inde par exemple, plusieurs fabricants locaux de téléphones portables ont su développer un avantage concurrentiel face aux géants du domaine, en inondant le marché de téléphones bon marché qui fonctionnent avec des batteries de très longue durée (plus de 2 semaines). Ces entreprises qui pratiquent des stratégies de coûts agressifs répondent toutefois aux besoins de populations pauvres avec un accès limité à l'électricité. Elles opèrent par simplicité dans la mobilisation des ressources et la réalisation des objectifs dès lors que les acteurs ont recours à l'informel, à des réseaux affinitaires, à diverses formes de solidarité mais aussi à des stratégies de contournement des 
contraintes (élimination des intermédiaires, entrepreneuriat effectual, prise en compte des « règles de terrain » et des « gens d'en bas », etc.).

Ensuite, le concept d'agilité est un construit plus ancien qui est souvent présenté comme une capacité pour une entreprise ou une organisation à «bouger avec justesse », c'est-à-dire à mieux affronter les difficultés et à saisir les opportunités issues d'un environnement de plus en plus instable et turbulent. Néanmoins, ce concept demande lui aussi à être contextualisé et opérationnalisé, voire revisité à l'aune d'une profonde transformation numérique selon Sambamurthy et al. (2003) tant les représentations des gestionnaires peuvent varier notamment sur ces dimensions de réactivité, de flexibilité, de rapidité, de technicité, de compétence et de talent. L'agilité est envisagée dans le modèle de l'entreprise africaine comme la capacité à se développer et à saisir des opportunités malgré des ruptures et des changements imprévisibles (émergence d'un virus mondialisé dévastateur, phénomènes climatiques, évolution de la mode ou même des mœurs, etc.). C'est une "capacité de mouvement pertinent individuel et collectif» (Barrand et al., 2018). En général, les auteurs parlent de stratégies frugales dès lors que ces entreprises africaines sont liées par des contrats implicites dans des contextes communautaires au sein desquels il n'y a pas de plans formels, de procédures écrites et une logique de pérennisation plutôt que de croissance. On relèvera aussi l'impact réel $\mathrm{du}$ profil psychologique du dirigeant sur la croissance des PME au Cameroun (Boubakary et al., 2017), en Côte d'Ivoire (Hernandez, 1997 ; Hernandez et Kamdem, 2007), au Bénin (Sogbossi, 2010) ou au Gabon (Ndoume Essingone, 2014 ; Mamboundou, 2009). Sur le plan de l'agilité, le dirigeant est souvent capable de saisir diverses opportunités, peut chercher à essaimer dans une autre ville (Libreville puis PortGentil et Franceville ou encore Oyem au Gabon en Afrique centrale par exemple) ou encore chercher à se diversifier en fonction des évolutions de l'environnement dans lequel il se trouve. Par exemple, on a pu observer l'évolution d'une Business School vers une transformation digitale avérée et maîtrisée. Sur le plan de la gestion des hommes, on parlera du modèle arbitraire agile de GRH (Nizet et Pichault, 2007) caractéristique d'un management paternaliste (Hernandez et Kamdem, 2007), d'une gestion discrétionnaire par préférence où le recrutement peut souvent se faire par parenté plutôt que par compétence, l'importance de la communauté créant des obligations familiales qui sont souvent une exigence communautaire mais qui fait toujours l'objet d'adaptation au fil de l'eau.

\section{2. «L'entreprise africaine » par une approche épistémologique et méthodologique singulière}

Sur le plan épistémologique, les recherches sur la gestion en Afrique s'inscrivent souvent dans des paradigmes très différents. À côté de travaux fondés sur une convergence internationale de la gestion et du management du fait de la globalisation, de nombreuses recherches adoptent une approche culturaliste, voire même proposent cet « autre chose » que nous cherchons à saisir à savoir la description d'un système cohérent de management africain. Certains auteurs débouchent sur la notion d'hybridité. D'autres travaux plutôt anglophones en 
sciences de gestion privilégient une approche dite « postcoloniale » tendant à souligner les effets de domination subis par le continent et proposent de fonder une épistémologie africaine seule à même de comprendre et d'appréhender les réalités locales (voir l'article de F.Y. Livian dans ce dossier).

Sur les plans méthodologique et empirique, il est également important de tenir compte des profondes spécificités africaines. Celles-ci créent en effet des conditions particulières à la recherche en gestion, à l'action, à l'observation et plus généralement à la collecte et à l'analyse des informations - faiblesse des données statistiques, informalité des règles, oralité dominante, cartographie des pouvoirs, manque d'accès au terrain, etc. - qui peuvent stimuler le déploiement de méthodes originales adaptées aux contextes. En cohérence avec plusieurs initiatives académiques appréhendant et discutant la gestion et le management en contextes africains (CAM 2017, CIL 2018 et 2019, ACIST 2018, AFAM 2018, AIMS 2019, AIM 2020, etc.), ce dossier s'interroge aussi implicitement sur la valeur des connaissances produites par les chercheurs sur les entreprises africaines. Il reste centré sur les alternatives africaines en management qui oscillent entre frugalité en termes de moyens pratiques et agilité en termes de modes d'action et d'appropriation. Par exemple, la gestion des entreprises et des organisations en Afrique - qu'elles soient formelles, informelles ou hybrides - fait l'objet de regards, de représentations et d'analyses parfois contradictoires.

- Pour les uns, les handicaps structurels, la domination des multinationales occidentales (et plus récemment encore chinoises, indiennes et asiatiques) ainsi que les spécificités culturelles africaines empêchent un vrai développement et enferment les entreprises africaines dans un rôle secondaire et marginalisé.

- Pour les autres, la forte croissance démographique et économique du continent, ainsi que ses richesses naturelles sont porteuses de grands espoirs et en fait un « eldorado des investisseurs » (Le Monde, 2012). Le continent accueille ainsi des entreprises souples et innovantes, au sein de sociétés « où se joue l'avenir du monde » (Mbembe, 2016).

- Pour beaucoup, sans nier ou sous-estimer les réelles poches de croissance et de réussites entrepreneuriales, il reste indispensable néanmoins de souligner le lancinant problème de la distribution des richesses créées localement et donc le peu de bénéfice qu'en tirent des populations locales qui restent encore largement rurales et précarisées (Jacquemot et Brunel, 2014). C'est pourquoi une approche pragmatique, concrète et ancrée, proche des réalités socioéconomiques, technologiques et managériales de l'organisation en Afrique permettrait probablement d'éviter les diagnostics parfois trop tranchés et de montrer la richesse, la diversité et la complexité de ces évolutions qui ont émergé récemment (Mbembe, 2016).

Plus spécifiquement encore, sur le plan méthodologique, cette forte activité créative impose aussi de repenser, de discuter et finalement d'élargir le modèle de management en Afrique, selon des modes spécifiques qui sauraient capitaliser sur les retours d'expériences en montrant l'inadaptation des pratiques et principes de gestion à l'occidentale aux particularités culturelles africaines. Par ailleurs, épistémologiquement parlant, il s'agit finalement de mettre 
en perspective théorique, conceptuelle, méthodologique et empirique, un management qui apparaît plus pragmatique et plus ancré et qui reste centré sur les acteurs, leurs réseaux, leurs stratégies, leurs projets, leurs perceptions et leurs représentations, leurs imaginaires, leurs dynamiques et, plus largement, sur des écosystèmes d'affaires. En définitive, les travaux de recherche menés en sciences de gestion sur, dans ou autour de « l'entreprise africaine », révèlent la diversité des problématiques traitées et même des cadres théoriques mobilisés (culturalisme, institutionnalisme, sociologie de l'action, de la régulation conjointe ou de la traduction, etc.), et aussi la richesse d'un champ de recherche assez aventureux et encore à investiguer.

\section{II - À LA RECHERCHE D'ALTERNATIVES AFRICAINES EN MANAGEMENT... QUELQUES PISTES}

L'intérêt croissant que présente le continent africain et les interrogations théoriques et conceptuelles qu'il soulève justifie l'engouement de plus en plus de chercheurs pour cette thématique. En ce sens, plusieurs perspectives et prolongement à ce dossier spécial semblent envisageables dès lors que l'on parle d'alternatives africaines en management.

\section{Enrichir l'analyse par une meilleure prise en compte de l'agir communautaire et des intelligences locales}

Afin de mieux appréhender la réalité de la société africaine et de son organisation sociale au sein de laquelle l'entreprise occupe une place significative, il convient de revenir aux enseignements des travaux fondateurs de Ferdinand Tönnies, sociologue et philosophe allemand (1855-1936) et à sa théorie des deux volontés. Pour Tönnies, l'unité de base de la vie en société est la relation sociale, ce qui implique une forte interdépendance entre les acteurs. Cette relation sociale est fondée sur la rencontre de deux volontés, la communauté et la société mais s'appuie sur une analyse fondée autour de types psychiques collectifs (Tönnies, 2010 [1912]). Pour Tönnies les rapports sociaux reposent sur des fondements psychiques. Ces rapports sont constitués de relations entre ce qu'il appelle des « volontés humaines », volontés envisagées comme l'ensemble des mécanismes qui motivent l'attitude des individus les uns à l'égard des autres. En ce sens, la volonté s'exprime de deux façons selon Tönnies ; une volonté organique (naturaliste) dans le sens où elle exprime directement un besoin vital, spontané voire impulsif et intuitif chez l'homme par opposition à une volonté réfléchie (domination de la pensée et de la raison sur les autres états de l'être). Volonté organique d'un côté (la passion, l'impulsion, le don, la générosité, etc.), volonté réfléchie de l'autre (le calcul, le rationnel, l'opportunisme, etc.), on le voit bien, l'apport de Tönnies est de montrer que ces deux volontés inspirent à l'activité humaine des conduites différentes sinon contradictoires pouvant conduire ainsi à deux types de rapports sociaux : les relations communautaires et les relations sociétaires. De façon générale, pour Tönnies, l'opposition de la communauté et de la société repose sur l'idée que le tissu social ne se résume pas seulement à de simples rapports contractuels entre les individus. D'autres liens, 
notamment familiaux, préexistent et perdurent. Ils ont leur raison d'être et demandent à être pris en compte. En ce sens, la notion des relations communautaires de Tönnies est éclairante pour mieux enrichir l'analyse de l'entreprise africaine dans le sens où elle se caractérise par la proximité des hommes, le partage des intérêts, la chaleur des rapports. Tönnies décrit ainsi un sentiment d'appartenance à la même collectivité qui domine à la fois la pensée et les actes des personnes. Fondée sur l'union et la coopération, la communauté inclut le sang (la famille, le clan, la parenté, et.), le lieu (voisinage, village, petites collectivités, etc.) mais aussi l'esprit (consensus autour d'idées ou de sentiments). Au total, la communauté est une organisation sociale obéissant à une logique bien précise et s'opposant d'un point de vue idéal typique à la logique sociétaire. Bref, l'individu n'existe pas au sein d'une communauté. Le clan, la famille, l'esprit de communauté sont des traits caractéristiques que les auteurs de ce dossier observent empiriquement en Afrique. Par exemple, ce qui se passe dans la famille, on le retrouve dans l'entreprise. Le chef de famille est aussi le chef d'entreprise et la forme verticale, hiérarchique, valorielle aussi est dominante dans l'entreprise africaine de petite taille. Cependant, la mondialisation et ses effets et l'influence de l'Occident (du «nord ») induisent aussi de nouveaux comportements : montée de l'individualisme, du calcul économique, de la recherche du profit, des comportements opportunistes, etc. En Afrique, le modèle de la communauté est aussi caractéristique du monde rural (éloignement des villes, le personnel des entreprises est souvent composite, soumis à de fortes contraintes locales, etc.). Généralement, on cherche à adapter avec une certaine agilité l'organisation du travail à la situation locale : répartition $\mathrm{du}$ travail par ethnie, roulement ethnique dans le recrutement des jeunes, distribution de la paye en espèces, etc. Sur le plan théorique, Livian (Livian et Reynaud, 2003) suggère souvent de mobiliser le cadre théorique de la régulation conjointe (Reynaud, 1989) qui convient bien pour montrer les tensions entre les règles officielles, formelles, qui viennent d'en haut et les règles autonomes (qui viennent $\mathrm{d}$ 'en bas) et les accommodements. Par ailleurs, on a aussi souvent observé des contournements, des ruses ou de séductions (stratégies d'acteurs) incompréhensibles vus du « nord » mais cohérentes du point de vue de l'intelligence des acteurs (souvent « les gens d'en bas ») et de leurs rationalités invisibles (voir l'analyse du cas Naomi dans Plane, 2008, 2019). En définitive, il semble que dans beaucoup d'entreprises communautaires, les règles « de terrain » l'emportent souvent sur « les règles officielles ».

\section{Considérer l'imaginaire, la spiritualité et leurs impacts sur les organisations africaines et les stratégies d'acteurs}

Avec le développement de réflexions sur les « dimensions oubliées » dans l'analyse des organisations (Chanlat, 1990), les questions de l'imaginaire et de la «vie psychique » font l'objet de travaux relativement récents (par exemple l'analyse en termes de «fantômes organisationnels », Bazin et Leclair, 2019). La sorcellerie et le mysticisme constituent des réalités pour les acteurs des organisations africaines. Comme le montre très bien dans ce dossier 
le texte de Biwolé-Fouda, l'imaginaire sorcellaire et, plus globalement, le mysticisme peuvent constituer des ressources idiosyncratiques qu'un entrepreneur par exemples au Cameroun, au Gabon, au Sénégal, au Bénin ou au Togo peut chercher à développer pour asseoir sa position concurrentielle compte tenu de son histoire personnelle, de sa spiritualité et de son vécu. La sorcellerie est omniprésente en Afrique subsaharienne (Kamdem et Tedongmo, 2015). Suivant ces auteurs, il est possible d'avoir en Afrique une lecture socioanthropologique du pouvoir dans l'organisation. En rapport étroit avec le monde invisible ou même mystique, l'univers de la sorcellerie est caractéristique du « système symbolique de croyances et de représentations collectives ». Certes, celui-ci est presque introuvable pour le chercheur en gestion. Cela dit, au Cameroun comme dans toute l'Afrique subsaharienne, la sorcellerie est une pratique courante mais " en parler, c'est déjà en faire » (Favret-Saada, 1977) ce qui explique une culture du silence, du secret et du sacré autour des pratiques sorcellaires, souvent envisagées comme des recours en vue de faire face à des oppositions, des concurrences, des compétitions ou même des conflits. L'organisation africaine semble constituer un lieu où la sorcellerie prend la forme «d'un art de mettre en scène les rapports sociaux» (Abéga et Abé, 2005). Dans son texte, Biwolé Fouda identifie plusieurs types de recours à la sorcellerie : le recours offensif (ensorceler), le recours défensif (désensorceler) et l'indifférence (neutralité). En résumé, il existerait des forces invisibles extérieures aux hommes et capables d'avoir une influence considérable sur leur devenir et sur la vie psychique de l'organisation
(Enriquez, 1992). Par ailleurs, la religion et la spiritualité occupent une place de plus en plus importante dans les recherches en sciences de gestion (Henley, 2016 ; Honore, 2014). Certains succès entrepreneuriaux s'expliquent par les impacts de la religion et des mouvements spirituels. Par exemple, Simen et Agne (2015) montrent que l'acceptation des pratiques religieuses contribuent à améliorer la productivité dans les entreprises sénégalaises. La prolifération en Afrique de mouvements spirituels assez variés, de la religiosité aussi s'expliquerait par la volonté des personnes de lutter contre des forces mystiques considérées comme particulièrement agissantes. Pour finir sur ce point, rappelons que l'organisation sociale africaine est complexe ; elle a ses propres codes, ses croyances profondes qui rejaillissent profondément sur le sociétal.

\section{Améliorer la compréhension de la culture pour mieux appréhender les modes de gouvernance et le management stratégique}

De nombreux travaux sur le fonctionnement des entreprises africaines insistent sur le paternalisme comme mode de gouvernance encore dominant. Celui-ci demeure aujourd'hui très caractéristique notamment s'agissant des petites organisations ; le chef d'entreprise est en même temps le chef de famille, de clan, etc. Il semble décider de tout... On retrouve un fonctionnement familial dans la plupart de ces entreprises en Afrique subsaharienne. La verticalité est liée à la structure de la société africaine où l'on va du plus petit au plus grand (importance de l'âge, du droit d'ainesse, etc.). Dans cette perspective, le chef d'entreprise prend inévitablement des 
décisions arbitraires, souvent discrétionnaires (il peut parfois faire preuve de favoritisme, etc.). Cependant, il n'existe pas une culture africaine homogène et identique mais des cultures africaines car l'environnement écologique, les traditions locales, les religions, l'expérience coloniale, le rapport à la modernité, etc. sont autant de facteurs qui ont façonné différemment les peuples africains (Kamdem, 2002). Si ce constat est vrai, il n'en demeure pas moins qu'il existe un substrat culturel commun aux peuples africains. Ainsi, les travaux d'Hofstede $(1980,2010)$ ont montré que les peuples africains tendent à être plus hiérarchisés, autoritaires, paternalistes et loyaux envers leurs groupes d'appartenance. Ouedraogo et Atangana-Abé (2014) ont analysé récemment les adaptations nécessaires à la culture africaine qui permettent de faciliter la compréhension de leur gouvernance ainsi que de leur gestion stratégique. Ces adaptions sont ainsi déclinées à travers six logiques : la logique de pouvoir, la logique de solidarité, la logique de la parenté symbolique, la logique de l'arbre à palabres, la logique polychronique et la logique fataliste. La logique de pouvoir consiste à revenir à ce qu'était l'Afrique traditionnelle : des communautés orales qui accordaient une importance capitale à l'expérience cumulée, où le patriarche était le plus ancien, le plus informé (Balandier, 1967). L'unité de base était la famille. Ainsi, quand des familles s'associaient et mettaient en commun leurs intérêts, elles formaient un village dont le chef était le premier parmi ses pairs. $\mathrm{Ce}$ dernier était chargé d'attribuer d'importantes responsabilités à ceux qu'il estimait aptes et jugeait de leur performance en dernière analyse (Asso, 1976). Finalement, il existait un chef traditionnel animiste ou islamisé qui exerçait un pouvoir sacré et surtout quasiment incontestable. La logique de solidarité s'apparente au mode de vie dans les villages en Afrique. C'est un schéma de régulation sociale avec un conseil des sages. Ce ne sont pas des individus avec qui vous avez affaire, mais des communautés avec des délégués communautaires. Les gens passent par ces relais pour être représentés. Cela instaure un rapport de force particulier qui se concilie mal avec la vision occidentale qui veut qu'un contrat ou un engagement soit passé à titre individuel. Au total, cette logique se manifeste par un système de droits et d'obligations que les membres de l'organisation ont les uns envers les autres. Il s'agit, pour le groupe, de l'obligation d'assurer collectivement la protection et l'épanouissement de chacun de ses membres. Elle contribue à assainir le climat social, à stimuler l'implication des employés et à favoriser une certaine productivité. Les salariés considèrent alors leur entreprise comme une seconde famille et la production de richesses comme un effort collectif (Ouedraogo et Atangana-Abé, 2014). La troisième logique est celle de la parenté symbolique. Que ce soit au Burkina Faso, au Cameroun ou au Bénin, la parenté symbolique - plus connue sous le nom de parenté à plaisanterie - est une pratique légendaire qui existe entre les ethnies, les clans et les individus de générations différentes. Elle peut parfois contribuer au déploiement de la stratégie dans la mesure où le dirigeant a su tisser des liens entre les membres de l'organisation qui ne sont pas nécessairement de nature biologique. Le rôle du dirigeant consiste alors à recréer au sein de son organisation cette forme de parenté en assimilant l'organisation à une grande famille 
qui transcende les liens de parenté réels entre certains de ses membres. En conséquence, beaucoup de choses entreprises doivent être inévitablement parrainées; la gestion et la sélection des personnes se font le plus souvent par parrainage (un réseau, une communauté, un frère, un parent, etc.). Plus particulièrement au sein des petites unités de production, les gens sont généralement adoubés par d'autres (logique de parenté symbolique).

La quatrième logique est celle de l'arbre à palabres. Palabrer est une coutume de rencontre et de création ou de maintien de lien social; la palabre est une véritable institution sociale à laquelle participe toute ou partie de la communauté d'un village. La logique de l'arbre à palabres est une coutume qui permet de régler un contentieux sans que les protagonistes ne soient lésés. En Afrique, on se réunit au pied de l'arbre à palabres pour discuter des décisions importantes à prendre pour l'avenir de la communauté (Chevrier, 2005). Finalement, on est presque dans la perspective d'un modèle discursif ou à la rigueur d'une « organisation dialogique » même si l'approche ici est plus verticale. L'arbre à palabres est une sorte de forum informel mais hiérarchisé où tous les problèmes de la cité se discutent sans tabou. Les sages écoutent, discutent et analysent les problèmes dans toutes leurs dimensions et prennent les décisions dans respect de la cohésion sociale du groupe (Bourgoin, 1984 ; Kessy, 1998). La logique polychronique correspond à une culture qui n'accepte pas bien d'être soumise au diktat du temps. Celui-ci n'est pas perçu comme devant être géré ou mesuré; il est néanmoins analysé en référence à des événements ou à des manifestations de la nature (Kessy,
2010) : les saisons, les épidémies, le chant du coq ou du rossignol, le lever du soleil ou son coucher, etc. Cette logique polychronique favorise l'adhésion et la recherche de consensus. Rien ne sert de précipiter les choses, de prendre une décision dans l'urgence en attendant que les conséquences suivent. Les acteurs au développement en Afrique et les réflexions autour de la gouvernance savent que sans patience, rien de concret et de productif ne peut se faire en Afrique (Musambi, 1996). Si l'on veut par exemple construire une école dans un village, et pour peu que la construction ne soit pas dans les normes de la région, il faudra longuement tenir une palabre pour obtenir l'intérêt, puis l'adhésion des villageois. De façon très empirique, on peut parfois avoir l'impression que les Africains donnent souvent l'impression de se mouvoir avec lenteur et en même temps de ne pas vraiment savoir anticiper. Pour finir, on peut aussi évoquer la logique fataliste (sixième logique). En effet, on peut rencontrer dans la culture de certains pays africains, des personnes qui utilisent l'expression «si Dieu le veut » ou « Inchallah ». À l'origine, ces expressions traduisent une humilité et une modestie face à la volonté de Dieu. Elle s'exprime ainsi : «je m'engage à réaliser quelque chose, tout en sachant que la volonté de Dieu est plus forte ». Malheureusement, cette attitude d'humilité a parfois été détournée au profit d'un fatalisme irresponsable. Ce dernier inhiberait l'esprit d'entreprise et saperait, par le biais de l'absence de responsabilité, la confiance mutuelle, deux attitudes essentielles pour le développement (Gendreau, 1998 ; Kabou, 1991 ; Ouedraogo et Atangana-Abé, 2014). On le sait, la culture en Afrique est un concept compliqué (Hounkou, 2015) et il 
existe certainement d'autres dimensions à explorer et à discuter. Par exemple, les dimensions sociales et relationnelles peuvent avoir de réels impacts sur le « système d'action concret » d'une organisation (la visite d'un beau-père peut amener le dirigeant à tout interrompre pour le recevoir, etc.). La division sexuelle du travail et le rôle particulier des femmes dans l'économie (l'entrepreneuriat féminin en particulier, Glidja, 2019 ou encore le rôle de la polygamie et des relations homme/femme, etc.) ont aussi des impacts considérables sur l'organisation sociale.

Cependant, les six dimensions mises en évidence permettent de mieux interpréter les modes de gouvernance et les styles de leadership parfois bien difficiles à décrypter sans ces codes (Nkakleu, Plane, Tchankam, 2017 ; Nyobe et Plane, 2020). En somme, ces réflexions indiquent qu'il y a certainement lieu d'intégrer le raisonnement de la contingence mais aussi de la synergique (Hampden-Turner et Trompenaars, 2000 ; Barmeyer, 2008) pour contribuer à l'amélioration des modes de gouvernance, de l'équilibre social à respecter et de la performance globale (Sogbossi, 2010). En effet, la synergie culturelle s'avère être l'approche appropriée pour tenter d'arrimer les divergences culturelles dans une démarche de management stratégique. Selon Barmeyer (2008), cette approche tente de prendre en considération les aspects positifs relatifs aux divergences culturelles afin de permettre un ajustement mutuel de valeurs opposées. Pour Hampden-Turner et Trompenaars (2000), cette approche de la synergie culturelle cherche à réconcilier des oppositions culturelles pour créer une valeur ajoutée à la rencontre interculturelle. Elle pourrait conduire à l'élaboration de modèles de gestion à valeur ajoutée en arrimant plus harmonieusement des valeurs a priori opposées comme celles du partage ou de la performance. Par exemple, il doit être possible de réconcilier la politique de rémunération basée sur des valeurs de partage avec les principes d'équité largement soulignés dans les modèles de management à l'occidentale ou encore de valoriser la socialisation des salariés par leurs relations de travail comme c'est parfois le cas au Cameroun (Kamning et al., 2020). De même, on doit être capable de réconcilier les valeurs liées au partage avec l'idée de la performance collective d'équipes de travail occidentales. De telles synergies peuvent mener à des solutions constructives au sujet de possibles oppositions culturelles, notamment dans le numérique (Bidan et al., 2020).

\section{Les contributions des auteurs à ce dossier}

Le texte proposé par Y.-F. Livian constitue une contribution qui expose l'intérêt et les limites de trois pôles théoriques utilisables pour situer une recherche en contexte africain. En premier lieu, le pôle culturaliste qui semble rester dominant et qui analyse les modèles de management africain comme hiérarchisés, paternalistes, ethniques, communautaires et spirituels. $\mathrm{La}$ personne est intégrée dans un ensemble plus ou moins cohérent dont elle n'est pas séparable. Ces modèles sont décrits comme fondé sur la solidarité, le collectivisme, la disponibilité et l'empathie. D'une certaine manière, les culturalistes évoquent un « modèle circulatoire » (Mutabazi, 2006) caractérisé par des échanges globaux symboliques, spirituels mais aussi économiques 
et sociaux dominés par la recherche du bien commun à la communauté. Livian évoque comme principale limite à ce modèle son essentialisme conduisant à élaborer des modèles fictifs n'ayant que peu d'emprise sur la réalité. Enfin, le courant de la « crossvergence » est évoqué comme une hybridation des convergences et des divergences culturelles, c'est-à-dire « une combinaison dynamique associant des forces socio-culturelles et l'idéologie générale $\mathrm{du}$ business international » même si les « transpositions managériales » semblent l'emporter sur les pratiques « indigènes ». En deuxième lieu, le pôle institutionnaliste exprime l'idée suivant laquelle les organisations africaines et leur gouvernance seraient ici façonnées par les normes, les règles, le système de formation, les lois et le droit international. Livian évoque à juste titre les travaux de Whitley (1999) et la notion de «business system » permettant de mettre en évidence les normes et valeurs dominantes, le rôle des États-nations, la structure de financement des entreprises, etc. L'idée principale est de monter l'encastrement du management à l'intérieur d'un cadre d'analyse sociopolitique plus large mais cette approche ne permet pas de bien saisir les idéologies, routines ou contraintes informelles davantage circulaires mais pouvant avoir un impact très fort sur les entreprises et les organisations. Enfin et en troisième lieu, Livian suggère un pôle postcolonial qui pense la recherche en contexte africain à partir d'une inégalité nord/sud et le retournement qu'elle devrait provoquer chez des penseurs ou des intellectuels cherchant des grilles d'analyse ou des « modes de pensée plus autonomes et d'égale valeur à ceux importés de l'extérieur et mieux adaptés aux contextes locaux ». Fondé sur le rejet des conceptions occidentales du monde, il y a lieu selon les tenants de ce courant de chercher d'autres approches plus « indéterminées » et davantage ancrées dans la conception africaine de l'homme en société. Ce courant postcolonial dans les recherches en management remet totalement la doxa des gestionnaires et vise à décoloniser des notions longtemps présentées comme universelles. Cependant, une telle approche est également problématique tant elle cherche à « essentialiser » les cultures et à minimiser l'impact du monde global sur les méthodes de management et la gestion. Par ailleurs, comme le souligne justement Livian, l'Afrique est un continent caractérisé par une dynamique de la mobilité et de la circulation et les représentations que les africains ont de leur entreprise et de leur mode de gestion sont inévitablement composites et le produit d'un « cosmopolitisme et des valeurs d'autochnie » (Mbembe, 2000 cité par Livian). Opposant d'une certaine manière Afrique et Occident, ce courant postcolonialiste pose enfin des problèmes épistémologiques quasiment insolubles car il s'agirait d'imaginer une science de la gestion qui ne serait que locale.

Au total, l'apport de Livian est de montrer la pertinence de ces trois pôles pour réaliser une recherche en contexte africain. Ces pôles présentent l'intérêt de mener à une approche approfondie du terrain africain, privilégiant une dimension collective et relativisant le poids des modèles. Ils s'opposent ainsi à une « vision technocratique de la gestion » et permettent une meilleure compréhension de ce qui se joue dans les rapports de force voire de domination ou encore dans l'analyse critique des écosystèmes d'affaires et des représentations qu'ils suscitent. Sur le plan 
méthodologique, l'auteur nous invite à mettre l'accent sur l'analyse de réalités locales notamment par des méthodologies qualitatives en mettant en avant la façon dont les africains gèrent le local et le global. Enfin, l'analyse critique des accommodements, des compromis ainsi que des « contournements et ruses » (Plane, 2000) permet de conceptualiser différentes formes d'hybridation des pratiques parfois inédites et sources d'équilibre social ou même parfois de performance dans une organisations. Bref, c'est en assumant le composite (Mbembe, 2016) ou même le «métissage » (Laplantine, 2001) que l'on pourra tirer des enseignements des expériences africaines afin qu'elle puisse devenir - dans le meilleur des cas - des alternatives africaines. Suivant Livian, la philosophie africaine pourrait à la fois être une contribution à la pensée critique (rejetant l'universalisme des modèles) et développant enfin une «pensée d'appartenances multiples » qu'il y a lieu d'assumer pour mener à bien ce type de réflexion.

Le texte de Geneviève Causse et de Jean Biwolé-Fouda développe les spécificités de ce que serait le modèle de l'entreprise africaine subsaharienne en particulier du point de vue de ses capacités de frugalité et d'agilité comme facteurs explicatifs de son dynamisme. Les auteurs fondent leur raisonnement sur un postulat : l'entreprise africaine existe. Partant de l'idée de Koanda (2005) suivant laquelle il coexisterait trois catégories d'entreprises: les entreprises contrôlées, les entreprises publiques et les entreprises communautaires, les auteurs vont s'intéresser aux entreprises qui relèvent souvent du secteur informel et qui sont ces entreprises communautaires même si les deux premières catégories peuvent inclure des pratiques traditionnelles ce qui peut aussi amener à parler des formes d'hybridation (Bakengela Shamba et Livian, 2014). Ils font alors l'hypothèse que l'Afrique est un continent relativement homogène au sein duquel il est possible d'élaborer un idéal type au sens de Weber de ce qu'est le modèle de l'entreprise africaine. L'entreprise africaine dont il est question ici présente les spécificités de gestion de la petite entreprise telles qu'elles ont déjà été bien conceptualisées (Marchesnay, 1991). En ce sens, on peut repérer dans la plupart des cas dans l'entreprise africaine « communautaire », une omniprésence du dirigeant-fondateur, une simplicité du système d'information et de gestion, un management de proximité, une absence de procédures ou du moins d'une bureaucratie ainsi que l'influence de l'âge du chef, de son milieu social d'appartenance et de son niveau d'éducation mais aussi de ses expériences passées et des variables psychosociologiques assez variées (besoin de réalisation de soi, besoin de pouvoir, aptitudes au leadership, etc.). L'entreprise africaine est alors envisagée comme étant fortement influencée par un contexte culturel précis à savoir le rôle de la communauté caractérisé par une appartenance à des groupes plus ou moins élargis comme la famille, le village ou l'ethnie. Ces appartenances créent des devoirs mais ne sont pas sans contreparties : elles apportent de la loyauté, de la confiance et le respect des ainés notamment. Au final, le chef d'entreprise agit tel un chef de famille voire, dans beaucoup de cas, tel un chef de village. Le mode de gestion en est fortement influencés. Les auteurs discutent du « comportement organisationnel » de l'entreprise africaine à partir des deux concepts privilégiés dans ce dossier spécial : la frugalité et l'agilité. En premier 
lieu, les auteurs développent le concept de frugalité qui se définit par l'idée qu'il est possible de « faire mieux avec moins ». En mobilisant les travaux de Radjou et Prabhu (2015) sur les innovations frugales, les auteurs développent aussi l'idée d'innovation inversée. La réflexion sur les innovations frugales et les observations que l'on peut faire en Afrique subsaharienne permettent de parler de la « débrouillardise » (« Jugaad » suivant le terme indien) dès lors que l'on est dans le cadre d'un produit moins cher que l'on met sur le marché et qui présente la caractéristique d'être fondé davantage sur «l'ingéniosité et le bon sens que sur le progrès technologique ». Les multiples observations des auteurs en contexte indiquent que l'on est bien dans le cadre d'une simplicité dans « la mobilisation des ressources et la réalisation des objectifs » dès lors que les agents économiques ont recours à diverses formes de solidarité et de logiques de contournement. En second lieu, le concept d'agilité est envisagé dans le modèle de l'entreprise africaine comme la capacité à se développer et à saisir des opportunités malgré des ruptures et des changements imprévisibles (émergence d'un virus dévastateur, phénomènes climatiques, évolution de la mode ou même des mœurs, etc.). C'est cette « capacité de mouvement permanent individuel et collectif » (Barrand et al., 2018). Les auteurs parlent de stratégies frugales dès lors que ces entreprises africaines sont liées par des contrats implicites dans des contextes communautaires au sein desquels il n'y a pas de plans formels, de procédures écrites et une logique de pérennisation plutôt que de croissance. Sur le plan de l'agilité, le chef d'entreprise est capable de saisir diverses opportunités, peut chercher à essaimer dans une autre ville ou encore chercher à se diversifier en fonction des évolutions de l'environnement dans lequel il se trouve. Sur le plan de la gestion des hommes, on parlera du modèle arbitraire de GRH (Nizet et Pichault, 2007) caractéristique d'une gestion par préférences où le recrutement peut souvent se faire par parenté plutôt que par compétences ; l'importance de la communauté crée des obligations familiales qui sont souvent une exigence communautaire forte. La place de la confiance est centrale dans ce modèle arbitraire car porteur de flexibilité dans le travail et d'attachement à l'entreprise. Sur le plan du marketing, les ventes de proximité sont souvent privilégiées ainsi qu'une approche très relationnelle. Dans les domaines de la comptabilité et de la finance, il n'y a pas vraiment de séparation entre le patrimoine personnel du dirigeant et le patrimoine de l'entreprise. Au final, l'accent est davantage mis sur la performance globale que sur des ratios rarement formalisés. On retrouve aussi l'approche de Mauss en termes de système de don et de contre-don avec la famille élargie voire parfois même le village. Sur le plan de la logistique, beaucoup de décisions sont intuitives et les transports peu onéreux et une gestion des flux (taxis) et des stocks par la débrouillardise est très courante. $\mathrm{Au}$ final, les auteurs avancent l'idée audacieuse d'une «théorie des organisations africaines » revisitée et qui reposerait sur sa propre rationalité. Celle-ci se justifie d'autant plus pour les entreprises typiquement africaines (communautaires) au sein desquelles les frugalités observées conduisent à beaucoup d'agilités dans les différents domaines de la gestion. Reste à poursuivre l'exploration de ce modèle et à 
l'enrichir notamment à l'aune des transformations numériques qui s'opèrent. Le texte de Jean Biwolé-Fouda et Henri Tedongmo Teko s'intéresse aux pratiques de sorcellerie en matière de management de la petite entreprise au Cameroun et présente le phénomène comme "une dimension oubliée » des recherches en management. Les auteurs se demandent dans quelle mesure les pratiques de sorcellerie influencent la dynamique concurrentielle de petites entreprises en contexte camerounais. À travers la prise en considération de variables irrationnelles, ils interrogent les concepts d'imaginaire sorcellaire et ses impacts sur les organisations en termes de dynamique concurrentielle. Ces pratiques sont généralement cachées et présentées comme culturellement ancrées. Elles pourraient fournir de nouvelles clés de lecture pour appréhender cette catégorie d'entreprises. L'originalité du texte réside bien dans la mise en relation entre l'univers rationnel de la dynamique concurrentielle et celui plus irrationnel de la sorcellerie. L'ensemble est présenté ainsi comme deux univers antinomiques n'ayant aucune relation entre eux. La sorcellerie est appréhendée dans le texte comme une « croyance selon laquelle le malheur inexpliqué est dû à l'intention maléfique d'individus dotés de pouvoir surnaturels » (Clément, 2003). D'une certaine manière, nous serions bien dans une sorte de management de l'invisible (une invisibilité du mal en l'occurrence ici) caractéristique du monde sorcellaire. Les auteurs présentent ce monde comme spirituel, fondé sur la puissance et destiné à rétablir un équilibre comportemental dans une logique de conquête et/ou de conservation du pouvoir et de privilèges. En ce sens, la sorcellerie est étudiée dans l'article comme un processus de domination quasiment au sens de Marx. Sur le plan théorique, la théorie des logiques d'action est mobilisée (Amblard et al., 1996) dans le sens où elle interprète les stratégies d'acteurs associées aux situations de gestion. Les auteurs mobilisent une méthodologie qualitative et s'appuient sur la méthode des cas. Trois minicas d'entrepreneurs camerounais sont ainsi analysés à partir de trois types de situation face aux pratiques sorcellaires (offensive/ défensive/neutre) et une enquête de terrain a été réalisée auprès de 60 entrepreneurs opérant dans les secteurs du textile et de l'habillement dans plusieurs marchés centraux au Cameroun. L'analyse des trois cas proposés par les auteurs indique bien que le marché est davantage un espace de rencontres entre dirigeants aux « trajectoires biographiques » variées ainsi qu'aux « imaginaires » réels. En ce sens, l'imaginaire sorcellaire apparaît comme une ressource idiosyncratique que l'entrepreneur développe pour asseoir sa position concurrentielle compte tenu de son histoire familiale, de ses références spirituelles et de son expérience passée. L'agir sorcellaire est une pratique qui semble courante mais en parler, c'est déjà en faire ce qui explique une culture du silence, $\mathrm{du}$ secret et du sacré autour de ces actions envisageés comme des recours en vue d'affronter des situations concurrentielles. Trois types de recours sont ainsi suggérés : le recours offensif (stratégie d'ensorceleur), le recours défensif (stratégie de désensorceleur) et l'indifférent (stratégie de neutralité). Finalement, la recherche proposée montre l'importance en Afrique subsaharienne d'un management par l'invisible mobilisant dans beaucoup de cas un monde magique, sacré davantage que la rationalité technico-économique. Les matériaux qualitatifs de 
Biwolé-Fouda et Tedongmo Teko montrent l'importance des pratiques magiques et spirituelles et leurs impacts dans l'univers très concurrentiel auquel sont confrontées les petites entreprises.

Le texte de Marielle A. Aka analyse la façon dont les indicateurs caractéristiques des mécanismes de clan se manifeste dans le contrôle de gestion de PME dirigées par des africains en Côte d'Ivoire. L'auteure s'appuie notamment sur le cadre d'analyse proposé par Ouchi pour développer l'idée que dans le mécanisme du clan, le contrôle formel est remplacé par une culture commune produisant une socialisation qui se manifeste notamment par un partage des valeurs. S'agissant de l'entreprise africaine, l'auteure parle de clan caractérisé par un ensemble d'acteurs développant des buts communs et un partage d'attentes en termes de rôles et de comportements (la loyauté étant une valeur dominante). Aka discute de la PME africaine en Côte d'Ivoire par son aspect communautaire mais aussi à partir d'une stratégie intuitive et peu formalisée. Opposant de façon un peu schématique, l'entrepreneur individualiste et rationnel occidental à l'entrepreneur communautaire africain (qui intègre une dimension sociale), l'auteure rappelle à juste titre les nombreuses solidarités ethniques, familiales, religieuse ou claniques traversées par ces PME ivoiriennes. $\mathrm{Au}$ fond, l'entrepreneur est avant tout un chef de famille et les relations d'emploi résultant souvent de liens de parenté. Dominés par des relations d'allégeance entre les ainés et les autres, les membres du clan se sentent redevables de leur emploi ; suivant cette logique normative, ils doivent en contrepartie obéissance, loyauté et respect. Suivant cette logique, la gestion du personnel est essentiellement discrétionnaire et revêt inévitablement un caractère arbitraire. L'analyse de l'enquête réalisée par l'auteure dans l'environnement ivoirien montre bien l'importance d'une gestion fondée sur la standardisation des valeurs et des normes de comportement. $\mathrm{La}$ méthodologie mobilisée est qualitative et le texte repose sur un échantillon de convenance de 40 entretiens réalisés auprès de dirigeants africains de PME ivoiriennes. L'analyse des résultats révèle la présence des six indicateurs caractéristiques des mécanismes du clan selon l'approche de Ouchi : procédures utilisées pour les recrutements, méthodes de suivi des activités, maîtrise des actions des employés, comportement au travail des employés, sources de motivations des employés et sources de satisfaction des dirigeants. Finalement, l'auteure suggère que le contrôle de gestion en Afrique est très fortement dépendant du dirigeant dans les PME ivoiriennes et que le mécanisme du clan constitue un moyen efficace pour la prise de décision et la réalisation des buts de l'entreprise.

Pour finir et avant de conclure, il y a certainement lieu d'élaborer un agenda de recherche peut être en vue d'un second dossier consacré aux alternatives africaines en management tant le terrain est fécond. D'abord, il faudra probablement tirer des enseignements des effets et des solutions locales imaginées en situation de management par les acteurs du développement de l'Afrique subsaharienne. Ensuite, des interrogations pourront être suscitées par les probables transformations des chaînes logistiques induites par la crise sanitaire provoquée par la pandémie de coronavirus et ses impacts sur les approches de la gestion des hommes et de structures. Enfin, il faudra certainement poursuivre la réflexion en 
cherchant à mesurer les effets de la transformation digitale en termes d'agilité et de frugalité en particulier et en essayant aussi de croiser les regards de chercheurs provenant d'univers disciplinaires variés (sciences de gestion, sciences humaines et sociales, santé, etc.).

\section{CONCLUSION}

Compte tenu du foisonnement des idées et des réflexions qui se développent considérablement ces dernières années (par exemple, AIMS, 2019 au Sénégal ou encore IAS, 2019 au Cameroun), ce dossier montre qu'il existe aussi parfois des divergences d'analyse assez importantes entre les chercheurs. $\mathrm{Ce}$ dossier consacré à « l'entreprise africaine : entre frugalité et agilité » vise à suggérer un agenda de recherche aux lecteurs de la revue et à encourager également les travaux sur des objets et des champs pouvant être divers (les TPE, les PME, les ONG, les églises, les hôpitaux, les grands groupes multinationaux et leurs logiques d'action, etc.). Cet article introductif visait aussi à rappeler les fortes contingences locales en Afrique subsaharienne: le management par l'ambiguïté, l'invisibilité de la gestion, l'importance des valeurs et du sacré, l'esprit de communauté au sens de Tönnies, la dimension mystique et spirituelle, la dimension sociale de l'entreprise, le rôle des clans, le rapport au temps et à la nature, les facteurs anthropologiques, religieux et l'ethnicité notamment. Nous soulignons l'idée également qu'il convient d'encourager l'entrepreneuriat effectual dans la mesure où l'aversion au risque en Afrique francophone semble constituer encore une contrainte assez forte. Au fond, le système est peu entrepreneurial probablement parce qu'il y a encore un manque de structures d'accompagnement entrepreneuriales. Les initiatives prises en ce sens sont bien entendu à encourager (par exemple, la création récente d'un incubateur d'entreprises à l'université de Lomé au Togo). L'hypermanagement du monde occidental est certes riche d'enseignements pour les chercheurs en gestion mais présente finalement bien des limites comme le montre certaines dérives de la mondialisation et de l'actualité pressante liée aux conséquences de la Covid-19. Cependant, l'hypomanagement - fondé sur une gestion implicite des choses et des personnes - que l'on peut rencontrer en Afrique témoigne aussi à la fois de la richesse d'un champ de recherche fécond et de l'intérêt à approfondir les investigations. Ces travaux mobiliseront probablement des approches et des méthodologies moins conventionnelles et plus ancrées dans des environnements locaux et dans des univers de sens variés.

\section{BIBLIOGRAPHIE}

Abéga S.C. et Abé C. (2005). « Approches anthropologiques de la sorcellerie », Justice et sorcellerie, edited by E. de Rosny, 33-47, Paris, Yaoundé: Karthala.

Amblard H., Bernoux P., Herreros G. et Livian Y.F. (1996). Les nouvelles approches sociologiques des organisations, Paris, Seuil. 
Asso B. (1976). Le Chef d'État africain. L'expérience des États africains de succession française, Paris, éd. Albatros.

Balandier G. (1967). Anthropologie politique, Paris, PUF.

Barmeyer C. (2008). « La GRH et les organisations face aux dynamiques culturelles », in Waxin M.-F., Barmeyer C. (coord.), Gestion des ressources humaines internationales. Problématiques, stratégies et pratiques, Paris, Éditions Liaisons, p. 57-102.

Bakengela Shamba et Livian Y.F. (2014). «Le management africain introuvable : Pour une approche de l'hybridité segmentée », Conférence ATLAS AFMI, mai, Marseille.

Barrand J.et al. (2018). Développer l'agilité en entreprise : De nouveaux leviers d'action et d'intelligence collective, ESF Sciences Humaines, $3^{\mathrm{e}}$ édition.

Bazin Y. et Leclair M. (2019). " "I see dead people..." À la rencontre des fantômes organisationnels qui hantent les entreprises », Revue française de gestion, vol. $45, \mathrm{n}^{\mathrm{o}} 283$, août-septembre, p. 11-29.

Bidan M., Biot-Paquerot G., Chaboud M. et Lentz F. (2020). «Inversion du domaine de l'adoption : les technologies latentes », Management \& Datascience, vol. 4, nº 2.

Biwolé Fouda J. (2015). « Essai de mesure de la performance sociétale des entreprises dans un contexte pauvre en informations non-financières: une approche par le mécanisme conciliateur », Revue Économie et Gestion, Cames, vol. 1, $\mathrm{n}^{\mathrm{o}} 1$.

Biwolé Fouda J., Causse G. et Ngantchou A. (2018). Théories des organisations africaines, Paris, L'Harmattan.

Boubakary B., Boukar H. et Tsapi V. (2017). « L'impact du profil psychologique du dirigeant sur la croissance des PME au Cameroun », Question(s) de management, vol. 2017/3, $\mathrm{n}^{\mathrm{O}} 18$, p. 47-62.

Bourgoin Henri. (1984). L'Afrique malade de management, Paris, Éditions Jean Picollec.

Chanlat J.-F. (1990) (éd.). L'individu dans l'organisation : les dimensions oubliées, Presses de l'université Laval, Québec.

Chevrier J. (2005). L'arbre à palabres. Essai sur les contes et récits traditionnels d'Afrique noire, Paris, Hatier International.

Clément F. (2003). «L'esprit ensorcelé. Les racines cognitives de la sorcellerie », revue Terrain, $\mathrm{n}^{\mathrm{o}} 41$ «Poésie et politique », septembre, p. 1-27.

Delalande P. (1987). Gestion de l'entreprise industrielle en Afrique, Paris, Economica.

Deschamps H. (1970). Les institutions politiques d'Afrique noire, $3^{\mathrm{e}}$ éd., Paris, PUF, coll. «Que sais-je ?», nº 549, pp. 13-36.

Enriquez E. (1992). L'organisation en analyse, Paris, PUF.

Favret-Saada J. (1977). Les mots, la mort, les sorts, Paris, Gallimard.

Fottorino E., Guillemin C. et Orsenna E. (1992). Besoin d'Afrique, Paris, Fayard. 
Gendreau F. (1998). «Pauvreté et changements démographiques », La Chronique du CEPED.

Godelier M. (2004). Métamorphose de la parenté, Paris, Fayard.

Glidja J (2019). «Les déterminants du succès de l'entrepreneuriat féminin au Bénin, le rôle modérateur de l'appui institutionnel : cas de la WBPC », Gestion 2000, vol. 36, n 2 , p. 39-59.

Giroux N. (1996). « La mise en œuvre discursive du changement », $5^{e}$ conférence de l'AIMS, Lille.

Hafsi H. et Farashashi M. (2005). "Applicability of management theories to developing

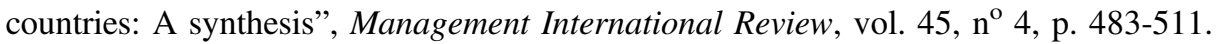

Hampden-Turner C. (1992). La Culture d'entreprise : Des cercles vicieux aux cercles vertueux, Paris, Seuil.

Hampden-Turner C. et Trompenaars F. (2000). Builiding Cross-Cultural Competencies, Chichester, Wiley.

Henley A. (2016). “Does religion influence entrepreneurial behaviour?”, International Small, Business Journal, July, p. 1-43.

Hernandez É.-M. et Kamdem E. (2007). « Universalité ou contingence de l'enseignement de la gestion Le cas de l'Afrique », Revue française de gestion, vol. 178-179, n ${ }^{\circ} 9$, p. $25-41$.

Hernandez E.-M. (1997). Le management des entreprises africaines, Paris, L'Harmattan, p. 146-147.

Hofstede G. (1980). Culture's Consequences: International differences in work-related values, Sage Publications, Beverly Hills.

Hofstede G.et al. (2010). Cultures et organisations, Paris, Pearson éditions.

Honore L. (2014). «Le management à l'épreuve de la religion », Revue Interdisciplinaire Management, Hommes \& Entreprises, vol. 4, n ${ }^{\circ} 13$, p. 54-67

Hoskisson R.E., Eden L., Lau C.M. et Wright M. (2000). "Strategy in emerging economies", The Academy of Management Journal, vol. 43, n 3, june, p. 249-267

Hounkou E. (2015). «Vers l'émergence de nouvelles valeurs culturelles en Afrique ? Etude des valeurs culturelles d'une population d'étudiants béninois et implications en matière de GRH », Revue Africaine de Gestion, numéro spécial.

Hugon P. (2006). «Les économies africaines dans la mondialisation », Problèmes économiques, numéro « Afrique : les chemins de la croissance », $\mathrm{n}^{\mathrm{o}} 29,13$ septembre.

Iribarne P. (d') (2003). Le Tiers-monde qui réussit : nouveaux modèles, Paris, éd. Odile Jacob. Jacquemot P. et Brunel S. (2014). «L'Afrique est-elle si bien partie ? , Afrique contemporaine, vol. $4, \mathrm{n}^{\mathrm{o}} 252$, p. 201-203.

Kabou A. (1991). Et si l'Afrique refusait le développement?, Paris, L'Harmattan 
Kamdem E. et Tedongmo T. (2015). «L'emprise sorcellaire en Afrique (Pouvoir et sorcellerie dans l'organisation en Afrique : une perspective interculturelle) », Revиe internationale de psychosociologie et de gestion des comportements organisationnels, vol. XXI, no 52 , p. 69-88.

Kamdem E. (1996). « Nouveau regard sur les pratiques du management au Cameroun », dans Organisation économique et cultures africaines : de l'homo oeconomicus à l'homo situs, Paris, L'Harmattan.

Kamdem E. (2002). Management et Interculturalité en Afrique: expérience camerounaise, Les Presses de l'Université Laval/L'Harmattan, Québec, Canada.

Kamdem E. (2010). "Itinéraire de recherche qualitative sur les temporalités en Afrique », Recherches qualitatives, $\mathrm{n}^{\mathrm{O}} 8$, p. 61-75

Kamning P., Nkakleu R. et Plane J.-M. (2020). «La socialisation des salariés par leurs relations de travail. Une étude de cas en contexte camerounais », Question( $(s)$ de Management?, $\mathrm{n}^{\mathrm{o}} 28$, juin, p. 17-34.

Kessy M.Z. (1998). Culture africaine et gestion de l'entreprise moderne, Paris, Édition CEDA.

Kessy M.Z. (2010). Renaissances Africaines, Paris, Éditions des ilots de résistance.

Kiggundu M.N., Jorgensen J.J. et Hafsi T. (1983). "Administrative theory and practice in developing countries: A synthesis”, Administrative Science Quarterly, vol. 28, p. 66-84.

Kiggundu M.N. (1989). Managing organizations in developing countries, Kumarian Press

Lalèyê Issiaka Prosper (1996). Organisations economiques et cultures africaines : de l'homo oeconomicus a l'homo situs, Paris, L'Harmattan.

Kabou A. (1991). Et si l'Afrique refusait le développement, Paris, L'Harmattan.

Koanda M. (2005). «Contribution à la connaissance du comportement financier et des performances financières des entreprises industrielles en Afrique : une application de la théorie de l'Agence », Thèse de doctorat en Sciences de Gestion, Poitiers.

Kouadio L.K. (2010). « La Solidarité africaine : les endettés sociaux », Courrier d'Afrique de l'Ouest, $\mathrm{n}^{\mathrm{o}} 73$, mars, p. 15-20.

Laplantine F. (2001). L'Anthropologie, Paris, Petite Bibliotheque Payot.

Livian Y.F. et Reynaud J.-D. (2003). " La théorie de la régulation conjointe », Encyclopédie des ressources humaines, Allouche J. et al., Paris, Vuibert, $3^{\mathrm{e}}$ édition 2012, p. 1968-1972.

Mamboundou J.P. (2009). « Du modèle arbitraire au modèle objectivant: quelques enseignements pour la théorie et la pratique de GRH, à partir de l'étude de cas de trois PME gabonaises », 20 congrès de l'AGRH, Toulouse, 5-6 novembre.

Marchesnay M. (1991). «La PME : une gestion spécifique », Économie rurale, Programme national Persée, $\mathrm{n}^{\mathrm{o}} 206$, p. 11-17.

Martinet A.-C. et Pesqueux Y. (2013). Épistémologie des sciences de gestion, Paris, Vuibert. 
Mbembe A. (2016). «L'identité n'est pas essentielle, nous sommes tous des passants », Le Monde, 25 octobre.

Monga C. (1993). « Le Mythe d'une gestion à l'africaine », Jeune Afrique Économie, nº 172, octobre.

Mutabazi E. (2006). «Face à la diversité des cultures et des modes de gestion : le modèle circulatoire de management en Afrique », Management \& Avenir, vol. 4, n 10, p. 179-197.

Mutabazi E. (2005). «Management de la diversité : l'enseignement africain », Business Digest, novembre, $\mathrm{n}^{\mathrm{o}} 157$, p. 9-10.

Musambi M.F. (1996). La conception du temps et développement intégré, Paris, L’Harmattan.

Mwissa K.C. (2005). Parenté et famille dans les cultures africaines, Yaoundé, Karthala.

Ndoume Essingone H. (2014). « Le poids des informations non financières dans la décision de crédit aux PME : une étude du cas gabonais », Techniques Financières et Développement, vol. $3, n^{\circ} 116$, p. 27-34

Nkakleu R. (2016). "Les pratiques de GRH des PME africaines sont-elles toujours informelles ? Une analyse contextualiste », Question(s) de management, $\mathrm{n}^{\circ} 12$, p. 83-104.

Nkakleu R., Plane J.M. et Tchankam J.P. (2017). « Leadership spirituel et renouveau du management ? », Réinventer le leadership, Caen, EMS Editions

Nizet J. et Pichault F. (2007). Les performances des organisations africaines : Pratiques de gestion en contexte incertain, Paris, L'Harmattan.

Nyambal E. (2008). Afrique : les voies de la prospérité : Dix clés pour sortir de la pauvreté, Paris, L'Harmattan, 2e édition.

Nyobe S. et Plane J.-M. (2020). "Hybrid HRM as a framework for handling cultural dominance issues at work", Revue de Gestion des Ressources Humaines, $\mathrm{n}^{\circ} 115$, janviermars, p. 5-18.

Ouchi W. (1982). Théorie Z, Paris, InterÉditions.

Ouedraogo A. et Atangana-Abé J. (2014). «La stratégie dans le contexte africain », Encyclopédie de la stratégie, Paris, Vuibert.

Ouedraogo A. (2004). Alliances stratégiques dans les pays en développement, spécificité, management et conditions de performance, Paris, éditions Publibook.

Pichault F. et Nizet J. (2000). Les pratiques de gestion des ressources humaines, Paris, Seuil, collection Points Essais.

Plane J.-M. (2019). Management des organisations. Concepts, théories, performances, Paris, Dunod, $5^{\mathrm{e}}$ édition.

Plane J.-M. (2008). «Séduction et management des hommes dans le contexte de l'hypermodernisme », Le Journal des psychologues, vol. 259, n 6, p. 49-53.

Plane J.-M. (2000). "The ethnomethodological approach of management: A new perspective on constructivist research", Journal of Business Ethics, vol. 26, n 3, august, p. 233-244. 
Radjou N., Prabhu J. et Ahuja S. (2013). L'innovation Jugaad, Redevenons ingénieux !, Paris, Eyrolles-Diateino.

Radjou N. et Prabhu J. (2015). Frugal Innovation: How to do More With Less, Paris, EyrollesDiateino.

Reynaud J.-D. (1989). Les règles du jeu. L'action collective et la régulation sociale, Paris, Armand Colin, $3^{\mathrm{e}}$ édition augmentée 1997.

Sambamurthy V.Bharadwaj A. et Grover V. (2003). "Shaping agility through digital options: Reconceptualizing the role of information technology", Contempory Firm, MIS Quaterly, vol. $27, \mathrm{n}^{\mathrm{o}} 2$, p. 237-263.

Sarasvathy S.D. (2001). "Effectual reasoning in entrepreneurial decision making: existence and bounds", Academy of Management Proceedings, AOM Meeting.

Simen S. et Agne A. (2015). « La gestion de l'entreprise sénégalaise : Entre ferveur islamique et laïcité problématique : quels impacts des pratiques religieuses dans l'entreprise sur le mode de gestion ? », SERGe Days, mai, Saint Louis, Sénégal.

Sogbossi B. (2010). "Perception de la notion de performance par les dirigeants de petites entreprises en Afrique », Revue des Sciences de Gestion, n 241. p. 117-124.

Tidjani B. et Kamdem E. (éd.) (2010). « Gérer les ressources humaines en Afrique : entre processus sociaux et pratiques organisationnelles », Caen, éditions EMS.

Tönnies F. (2010) [1912]. Communauté et société. Catégories fondamentales de la sociologie pure, Paris, PUF, première édition française 1912.

Whitley R. (1999). Divergent Capitalisms: The Social Structuring And Change Of Business Systems, Oxford, Oxford University Press.

Worou D.R. (2011). «La sagesse polyphonique : trois cas de pilotage de changement organisationnel dans des entreprises d'Afrique », Revue @GRH, vol. 1, n 1, p. 73-100. 